\section{$1 N-89$}

NASA Technical Memorandum 88368

61188
Airborne Astronomy Program Medium Altitude Missions Branch Preprint Series 063

(KASA-TM-8836E) TEE ICNIZATICN STRUCTURE OF

TEE OAICN HEECLA: INEEARED IINE CESERVATICNS

N87-19313

ADC DCDELS (NASA) $46 \mathrm{~F}$

CSCL O3A

$63 / 89 \quad \begin{array}{ll}\text { Unclas } \\ 43769\end{array}$

\title{
The Ionization Structure of the Orion Nebula: Infrared Line Observations and Models
}

J.P. Simpson, R.H. Rubin, E.F. Erickson, and M.R. Haas

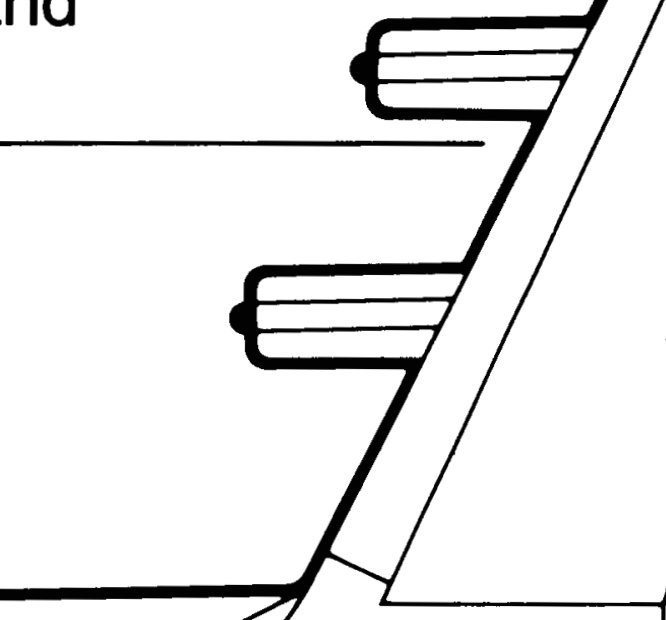

October 1986
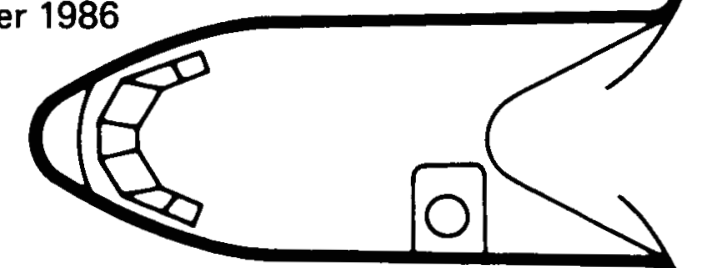


\section{The Ionization Structure of the Orion Nebula: Infrared Line Observations and Models}

J. P. Simpson, Lick Observatory, University of California, Santa Cruz, Califronia

R. H. Rubin, University of California, Los Angeles, California

E. F. Erickson, Ames Research Center, Moffett Field, California

M. R. Haas, Mycol, Inc., Sunnyvale, California

October 1986

\section{N/Sก}

National Aeronautics and

Space Administration

Ames Research Center

Moffett Field, California 94035 


\section{ABSTRACT}

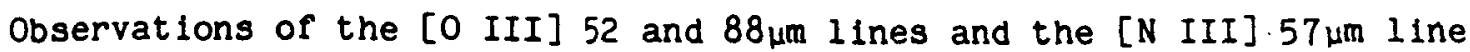
have been made at 6 positions and the [Ne III] $36 \mu \mathrm{m}$ line at 4 positions in the Orion Nebula to probe its ionization structure. The measurements, made with a -40" diameter beam, were spaced every 45" in a line south from and including the Trapezium. The wavelength of the [Ne III] line was measured to be $36.013 \pm 0.004 \mu \mathrm{m}$. Electron densities and abundance ratios of $\mathrm{N}^{++} / 0^{++}$have been calculated and compared to other radio and optical observations. Detailed one component and two component (bar plus halo) spherical models were calculated for exciting stars with effective temperatures of $37-40,000 \mathrm{~K}$ and $\log \mathrm{B}=4.0$ and 4.5 . Both the new infrared observations and the visible line measurements of oxygen and nitrogen require $T_{\text {eff }} \leq 37,000 \mathrm{~K}$. However, the doubly ionized neon requires a model with $\mathrm{T}_{\text {eff }} \geq 39,000 \mathrm{~K}$, which is more consistent with that inferred from the radio flux or spectral type. These differences in $T_{\text {eff }}$ are not due to effects of dust on the stellar radiation fleld, but are probably due to inaccuracies in the assumed stellar spectrum. The observed $\mathrm{N}^{++} / 0^{++}$ratio is almost twice the $\mathrm{N}^{+} / 0^{+}$ratio. Our best $\mathrm{fit}$ models give $\mathrm{N} / \mathrm{H}=8.4 \times 10^{-5}, 0 / \mathrm{H}=4.0 \times 10^{-4}$, and $\mathrm{Ne} / \mathrm{H}=1.3 \times 10^{-4}$. Thus neon and nitrogen are approximately solar, but oxygen is half solar in abundance. From the infrared $0^{++}$lines we conclude that the Ionization bar results from an increase in column depth rather than from a local density enhancement.

Subject Headings: Nebulae: H II Regions - Nebulae: Orion Nebula Nebulae: abundances 


\section{INTRODUCTION}

H II regions are studied in order to learn about interstellar elemental abundances and the far ultravlolet spectra of hot stars. The Orion Nebula is a case to study because it is nearby, bright, reasonably compact, the extinction is not large in the visible and is negligible in the infrared. There are radio maps avallable at high spatial resolution for determining the electron density. It has long been studied in the radio and visible, but only recently in the UV and infrared.

Infrared forbidden lines are important in an observational study of the structure of an H II region because some of them arise from lonization states that do not have optical forbidden lines. Such lines include transitions of $\mathrm{N}^{++}$ at $57 \mu \mathrm{m}, \mathrm{C}^{+}$at $158 \mu \mathrm{m}, \mathrm{Ne}^{+}$at $12.8 \mu \mathrm{m}, \mathrm{S}^{+++}$at $10.5 \mu \mathrm{m}$, and $\mathrm{Ar}^{+}$at $6.98 \mu \mathrm{m}$. Additional advantages are that the lines are less affected by extinction than are the optical lines and not at all by scattering. Since infrared forbidden lines (unlike optical) are insensitive to electron temperature, the abundances determined from them depend weakly on the temperature determination. Ions with lines in both the infrared and the visible include $\mathrm{O}^{++}, \mathrm{Ne}^{++}, \mathrm{S}^{++}$, and $\mathrm{Ar}^{++}$.

One Important study at optical wavelengths was done by Osterbrock and Flather (1959), who compared the intensities of HB, [O II] 3726 and $3729 \mathrm{~A}$, and [O III] $5007 \mathrm{~A}$ out to a distance of 30 arc min from the exciting stars in the Trapezium. Spherically symmetric thermal and Ionization equilibrium models such as those by Rub in (1968, 1985) and Simpson and Rubin (1984) (hereafter SR) do not reproduce these observed line ratios; that is, the ratios predicted from the models imply too few singly ionized lons in the center and too few doubly lonized lons at great distances from the exciting stars. The latter can be explained by the 
fact that the Orion Nebula is also a reflection nebila, as can be seen from continuum observations. In fact, Peimbert (1982) finds that all optical lines seen farther than about 12' from the Trapezium are produced entirely by scattered light. However, due to a central hole in the dust distribution (O'Dell and Hubbard 1965, Simpson 1973, Schiffer and Mathis 1974), the inner several arc min of the nebula are negligibly affected by scattering (Peimbert 1982).

Another reason for studying the Orion Nebula is that the highly obscured, compact H II regions that have been modeled by Zellik (1977), Herter, Helfer, and Pipher (1983), Rubin, Hollenbach and Erickson (1983) (hereafter RHE), and SR do not show the high ionization that is predicted for the hot massive stars required to match the luminosities deduced from radio observations. Possible solutions to this discrepancy are that the H II regions are excited by clusters of stars (Lacy, Beck, and Geballe 1982, RHE, SR), the Ionized gas is mixed with dust that softens the stellar spectrum (Herter, Helfer, and Pipher 1983), or that the model stellar atmospheres used for the computations do not provide realistic representations of the Lyman continuum as a function of frequency and stellar effective temperature $T_{\text {eff }}$ (RHE and Abbott and Hummer 1985). Because the Orion Nebula is nearby and relatively unobscured, we can see the exciting stars and directly determine their spectral types. It is excited predominantly by the $06 \mathrm{p}$ star $\theta^{1}$ Ori $C$ (Lee 1968) and several 09.5 stars that provide only a small fraction of the total flux. An 06 star supposedly has $\mathrm{T}$ eff $=40,000 \mathrm{~K}$ (Cont 1 1973), although more recent work (Pottasch, Wesselius, and van Duinen 1979) prefers a lower $T_{\text {eff }}(-38,000 \mathrm{~K})$ for this spectral type. Mathis (1985) has already suggested that $\mathrm{T}_{\text {eff }}$ should be lower than $40,000 \mathrm{~K}$ for Orion, based on the $0^{+} / 0^{++}$ratio. We know that there is not enough dust to affect the ionizing 
spectrum in the center where there is the hole in the dust distribution. Comparison of new models of the Orion Nebula with the extensive infrared and optical observations should elucidate whether the deduced low ionization is a general problem, or whether it is confined to obscured dusty $H$ II regions.

Another problem we wish to address is the abundance of nitrogen relative to oxygen. Because nitrogen and oxygen have similar ionization potentials, Peimbert and Costero (1969) assumed that their ionization structures are identical and that one could correct for the missing $\mathrm{N}^{++}$in abundance studies of H II regions by multiplying $\mathrm{N}^{+} / \mathrm{H}^{+}$by the ratio $\left(\mathrm{O}^{+}+\mathrm{O}^{++}\right) / \mathrm{O}^{+}$. Peimbert and Torres-Peimbert (1977) (hereafter PTP) and Torres-Peimbert, Peimbert, and Daltabuit (1980) (hereafter TPPD) studied optical lines throughout the or ion Nebula to obtain an $\mathrm{N}^{+} / 0^{+}$ratio of 0.10 , which they also give as the $\mathrm{N} / \mathrm{O}$ ratio. However, a line from $\mathrm{N}^{++}$can now be observed in the far infrared at $57 \mu \mathrm{m}$, as can lines of $\mathrm{O}^{++}$at 52 and $88 \mu \mathrm{m}$. These three lines are mainly density sensitive (the optical lines are more sensitive to temperature), but the density can be determined from the [O III] 52/88 ratio if the lines are optically thin, which they are in all but the largest H II reglons (Rubin 1968, Simpson 1975). Lester et al. (1983) in their study of these three infrared lines in several H II regions found that the $\mathrm{N}^{++} / 0^{++}$ratio is typically 0.2 or higher, and is 0.30 for the radio peak of the Orion Nebula itself!

$\mathrm{N}^{+}$does have a lower ionization potential $(29 \mathrm{eV})$ than $\mathrm{O}^{+}(35 \mathrm{eV})$, and in our $H$ II region models (RHE; SR; Rubin 1985) the $\mathrm{N}^{++}$lonization zone is larger than the $\mathrm{O}^{++}$zone (except for the dense regions with $\mathrm{T}_{\text {eff }}>40,000 \mathrm{~K}$ ). Moreover, in regions excited by lower temperature stars and in regions where the matter is clumped, the ratio $\mathrm{N}^{++} / \mathrm{O}^{++}$inferred by the technique of Lester et al. (1983) is 
considerably larger than the input $N / O$. For 06 stars like $\theta^{2}$ Ori $C$ with $\mathrm{T}_{\text {eff }}-40,000 \mathrm{~K}$, the $\mathrm{N}^{++}$zone is similar to the $\mathrm{O}^{++}$zone in a nebula of uniform density (Rubin 1985). However, the models show that the lower the effective temperature of the exciting star, the smailer the $\mathrm{O}^{++}$region compared to the $\mathrm{N}^{++}$ region. Thus high spatial resolution observations should distinguish between an enhanced [N III ]/[0 III] ratio due to (a) a larger $\mathrm{N}^{++}$zone than the $\mathrm{O}^{++}$zone, or (b) some other factor such as different collisional excitation cross sections.

In this paper, (1) we examine the $\mathrm{N}^{++} / 0^{++}$ratio as a function of position in the nebula to see if it changes as the models predict. (2) We test the previous assumption that the optical line strengths are not affected by scattering in the interior several arc minutes by comparing the optical and infrared [O III] and [Ne III] intensities. (3) We investigate the problem of the overall degree of Ionization being lower than predicted from the spectral type of the exciting star observed or inferred from the radio flux - a problem that was first discovered in highly obscured compact $H$ II regions.

An investigation like this is best carried out by comparing observations and predictions of lines from several different elements with significantly different ionization potentials (such as nitrogen, oxygen, and neon). To do this in a reasonable amount of observing time, we chose not to map the entire Orion Nebula, but to observe only one sector. To be sure we had a complete sample of the ionization structure as a function of distance from the exciting star, we observed in a straight Ilne away from the Trapezlum. We chose the strip with the largest number of optical observations so that we could compare our detailed theoretical models to the singly lonized elements that have only 
optical 1ines. We also wanted to avold the separate $H$ II regions around the $\theta^{2}$ Ori stars (Pelmbert 1982). The final cholce was a line due South from the Trapezium. This included the ionization front of the bar, which we had to model as a shell of higher density, since our models are spherically symmetric. The simplification of observing and modeling only one sector has the advantage that we are not forced to average over the whole nebula; thus the chemical composition and stellar effective temperature herein derived should not be affected by the limited solid angle of the investigation.

The remainder of the paper is divided as follows: In the next section we present new observations of the far infrared transitions of $\mathrm{O}^{++}, \mathrm{N}^{++}$, and $\mathrm{Ne}^{++}$ in Orion. We then introduce our detailed theoretical models of the nebula in Section III. In Section IV the models are compared with our data and other published data in a detalled way. Our major conclusions are summarized in Section $V$.

\section{OBSERVATIONS AND DATA ANALYSIS}

The observations were carried out with the $91 \mathrm{~cm}$ telescope of the Kufper Alrborne Observatory on 1983 November 21 and 1985 February 4, 6, 12, and 14 using the facllity cooled grating spectrometer (CGS) described by Erickson et al. (1984a, 1985). The aperture profiles, which were measured in the laboratory using a small blackbody source, had a FWHM of $37^{\prime \prime}$ in 1983 November and $46.5^{\prime \prime}$ in 1985 February. In both cases the beam's effective area corresponded to an equivalent disk -1.04 as large, or $2.7 \times 10^{-8}$ ster in 1983 and $4.3 \times 10^{-8}$ ster in 1985. The chopper throw was $4^{\prime}$ in azimuth (approximately $E-W$ ). Sequences of four 5 or $10 \mathrm{~s}$ integrations were taken with the source placed alternately in 
right and left beam to correct for linear variations in the background flux. In 1983 November the [O III] 51.8 and $88.4 \mu \mathrm{m}$ lines and the [N III] $57.3 \mu \mathrm{m}$ line were observed at the 6 spatial positions (P1-P6) given in Table 1. In 1985 February the same three lines were reobserved with improved signal-to-noise at positions $\mathrm{P1}, \mathrm{P4}, \mathrm{P5}$, and $\mathrm{P6}$; the [O III] $51.8 \mu \mathrm{m}$ line was observed at $\mathrm{P7}$, and the [Ne III] 36.0 $\mathrm{\mu m}$ line was observed at positions $\mathrm{P} 1-\mathrm{P} 4$. The 1985 observations are also summarlzed in Table 1.

The positions observed lie in a line starting at $\theta^{2}$ Ori $C$ and running stralght south. Absolute positions were determined to $\pm 5^{\prime \prime}$ by offsetting from elther $\theta^{1}$ Or $1 \mathrm{C}$ or $\theta^{2}$ ori $A$. The infrared boresight was verifled in elight by assuming that the $63.07 \mu \mathrm{m}$ continuum peaks at the KleInmann-Low nebula (KL) at R.A. $(1950)=5^{h} 32^{m_{4}} 46.7^{3}, \delta(1950)=-5024^{\prime} 27^{n}$ (Werner 1982). The total bandpass of the six Ge:Ga photoconductor detectors was sufficlent to include both the line and the adjacent continuum using a single grating position. At the different wavelengths observed the spectral resolving power was $3300-4200$ in 1983 November and 2600-3300 in 1985 February.

On the 1983 November flight the absolute flux calibration and the relative detector responses were determined by observing the Mars continuum at each wavelength. The final reduced spectra are the signals obtained at the map positions divided by the signals obtained on the Mars continuum, multiplied by the flux from Mars as computed using the model of Simpson et al. (1981), and corrected for the difference in the water vapor column density. The line-of-sight water vapor was measured to be 16 precipitable microns towards Mars and 11 precipitable microns towards $\mathrm{KL}$ by observing a water line at $84.42 \mu \mathrm{m}$. Since there are no strong features in the vicinity of these lines, the 
resultant correction to the data was $55 \%$. The [O III] line fluxes were increased by $13 \%$ because of a synchronous demodulation phase adjustment between them and the Mars calibration.

In 1985 February the absolute flux calibration and the relative detector responses were determined by observing $\mathrm{KL}$ on each flight in the cont inuum adjacent to the lines. Calibration spectra taken before and after the mapping observations on a single flight typically agreed to better than $\pm 5 \%$. The change in the instrument function between these nearby wavelengths was determined to $\pm 5 \%$ using an onboard calibration source. On 1985 February 4 and 6 the line-of-sight water vapor towards $K L$ was measured to be 9 precipitable microns by observing water lines at 60.33 and $64.22 \mu \mathrm{m}$. This water vapor value was assumed for 1985 February 12 and 14 as well. The water vapor correction was $15 \pm 8 \%$ for [Ne III] $36.0 \mu \mathrm{m}$, which sits on the shoulder of a relatively strong telluric water line, and $55 \%$ for the [O III] and [N III] lines. The final reduced spectra are the signals obtained at the map positions divided by the corresponding signals obtained on the KL continuum and multiplied by (a) the ratio of two water vapor spectra to correct for the difference in transmission, (b) the change in the instrument function, and (c) the continuum flux from KL as given by Erickson et al. (1981). At both epochs the effect of diffraction in the telescope was computed to be $\$ 10 \%$ and, hence, was ignored. We estimate the overall uncertainty in the absolute calibrations to be $\pm 25 \%$. The agreement between the two dates is consistent with this considering the different beam sizes.

The line strengths in Table 1 are the result of a nonlinear least squares fit to a flat continuum level and a superimposed Gaussian line profile 
integrated over a detector bandpass. The four free parameters for the fitted curves are the line width, line height, line position, and continuum level. The line width provides a measure of the instrumental resolution since the lines are unresolved; the line position provides an estimate of the uncertainty in the wavelength callbration. For the weaker lines the width and/or position were assumed known and the line and continuum strengths were fitted. The error bars quoted in Table 1 are one-standard-deviation-of-the-mean statistical errors. The additional uncertainty in the ratio of line strengths due to systematic effects is estimated to be roughly half the absolute uncertainty quoted above for the individual line strengths.

The [Ne III] 36.0 $\mathrm{mm}$ line was first detected by Erickson et al. (1984b) in M17 and subsequently in the planetary nebula NGC 6543 by Shure et al. (1984). The present Orion observations at positions P1-P4 are displayed in Figure 1. The line at P2 was observed using two nearby grating positions to obtain a better measurement of the continuum. From the present orion data $\left(\mathrm{V}_{\mathrm{LSR}}=-3.5 \mathrm{~km} \mathrm{~s} \mathrm{~s}^{-1}\right.$ ) and new observations of $M 17\left(V_{L S R}=18 \mathrm{~km} \mathrm{~s}^{-1}\right)$, we find the rest wavelength of the line to be $36.013 \pm 0.004 \mu \mathrm{m}$, in agreement with our original detection.

From the [O III] $52 / 88 \mu \mathrm{m}$ line ratio, we have derived the electron densities $\mathrm{N}_{\mathrm{e}}$ in Table 1 (c.f. Lester et al. 1983) using the collisional excitation cross sections of Aggarwal, Baluja, and Tully (1982). Using these densities and the $57 / 52 \mu \mathrm{m}$ ratio, we derived the values of $\mathrm{N}^{++} / 0^{++}$listed in Table 1 . With the most recent cross sections for $\mathrm{O}^{++}$and $\mathrm{N}^{++}$(Nussbaumer and Storey 1979), the $57 / 52 \mu \mathrm{m}$ ratio is not very sensitive to density; the error bars for $\mathrm{N}^{++} / 0^{++}$are those of the $57 / 52 \mu \mathrm{m}$ line strength ratio. As predicted by the models discussed below, the $\mathrm{N}^{++} / 0^{++}$ratio does increase slightly with distance from the Trapezium 
(position P1). The agreement of our data with that of other observers such as Lester et al. (1983) and Furniss et al. (1983) is adequate, considering that either our spatial positions do not agree or our bean sizes are quite different.

\section{MODELS OF THE ORION NEBULA}

For objects like the Orion Nebula that have a large range of electron densities and where different lines of sight sample different lonization zones, one can best study the ionization structure and chemical composition by comparison with detailed models. Our models do not include far UV or radio recombination lines, but focus on the physics of the infrared and optical lines.

a) Spherical Models

We first consider traditional spherically symetric models. Those computed here use the H II region model program described by Rubin (1968) and updated by Rubin (1983) and SR. The electron densities of the models are those that reproduce the radio brightness maps at the same positions as our measurements (Table 1) and extending further south. The models are intended to reproduce only the observations along our cut south from the Trapezium. In Figure 2 we have plotted small beam radio brightness temperatures and the $H \beta$ measurements of PTP scaled to $5 \mathrm{GHz}$ for a line south from the Trapezium. There are two curves for Martin and Gull (1976) - the solid line is south from the Trapezium and the dashed line is south from the radio peak ( $\leq 20^{\prime \prime}$ west of $\theta^{1}$ Or $1 \mathrm{C}$ ). Their beam extended 20" in the north-south direction, and shows the bar clearly. A curve for the VLA map of Johnston et al. (1983) (4.8 GHz; $16^{\prime \prime} \times 13^{\prime \prime}$ beam) is also plotted. The absolute flux levels are substantlally lower, no doubt because the 
VLA is not sensitive to smooth extended emission. However, the general structure of the peak and bar are very similar. The map of Rodriguez and Chaisson (1978) (23.4 GHz; 80" beam) shows the sharp fall-off beyond the bar but none of the structure within the bar. The map of Wilson and Pauls (1984) (also $23 \mathrm{GHz}$; 43" beam) is similar. The other maps were made by Johnston and Hobbs (1969) (1.6' beam; 9.55mm) and by Schraml and Mezger (1969) (2' beam; $1.95 \mathrm{~cm}$ ). These last 4 maps show the outer structure of the $H$ II region that the interferometer maps do not, but they are badly degraded by their poor resolution within about 4' from the Trapezium. The composite radio map that was used in the models reproduces the high radio peak (curve 1 for distances $50.25^{\prime}$ ), the trough and bar of curve 2 out to $2.25^{\prime}$, and then decreases slowly from $\mathrm{I}_{B}-60 \mathrm{~K}$ at $2.25^{\prime}$ to meet the large beam fluxes at 4.5'. (The "trough" is the lower intensity region at -1 '; the "bar" is at 1.5-2.0'.) The high density maximum at the Trapezium in the model density distribution was used because our $37^{\prime \prime}$ and 46.5" beams include much of the radio peak, even when centered on the Trapezium

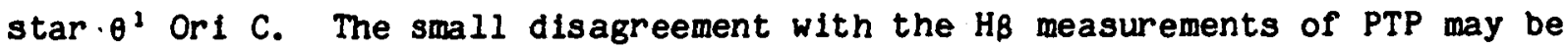
due to the uncertainties in the extinction correction (approximately one magnitude) or to beam size effects. The resultant density distribution adopted for the spherically symmetric one component model is shown in Figure 3, which includes the effects of clumping as discussed below.

The physics of the models is the same as that used by SR with the addition of the new collisional excitation cross sections for [Ne II] and [Ne III] calculated by Bayes, Saraph, and Seaton (1985) and by Butler and Mendoza (1984), respectively, and new charge transfer cross sectlons for $\mathrm{O}^{++}+\mathrm{He}^{-0}+0^{+}+\mathrm{He}^{+}$by Butler, Hell, and Dalgarno (1984). Again we used the line-blanketed LTE model atmospheres of Kurucz (1979) for effective temperatures of $40,000 \mathrm{~K}$ 
$(\log g=4.5)$ and $37-40,000 \mathrm{~K}(\log g=4.0)$. The luminosities of the stars exciting the models were adjusted to give a Stromgren radius of about 8 arc min, which corresponds to the slze indicated by the radio maps; the resulting Lyman continuum flux is $10^{49}$ photons $\mathrm{s}^{-1}$. The radius of the exciting star for each model was adjusted accordingly; they range from $10.2 R_{\odot}$ for $T_{\text {eff }}=40,000 \mathrm{~K}$ to $13.8 R_{\odot}$ for $T_{\text {eff }}=37,000 \mathrm{~K}$, both with $\log 8=4.0$. Models with $T_{\text {eff }}=39,000 \mathrm{~K}$ and $108 \mathrm{~g}=4.0$ are very similar to the models with $\mathrm{T}_{\text {eff }}=40,000 \mathrm{~K}$ and $\log \mathrm{g}=4.5$. Since our method determines abundances by comparing model predictions to observations, we beg in with abundances taken from the 11 terature: $\mathrm{He} / \mathrm{H}=0.10, \mathrm{C} / \mathrm{H}=3.3 \times 10^{-4}$, $\mathrm{N} / \mathrm{H}=4.5 \times 10^{-5}$, and $\mathrm{Ar} / \mathrm{H}=4.5 \times 10^{-6}$ (PTP and TPPD) and $\mathrm{O} / \mathrm{H}=4.0 \times 10^{-4}, \mathrm{Ne} / \mathrm{H}=8.1 \times 10^{-5}$, and $S / H=2.2 \times 10^{-5}$ (Lester, Dinerstein, and Rank 1979, hereafter LDR). We WIIl discuss the uncertainties in these abundances later. For spherical models the gas must be clumped, since the observed line ratios that are sensitive to density imply much higher densities than implied by the computed ratios for models without clumping. We define the clumping factor as the inverse of the filling factor. A clumping factor of 4.0 was derived by considering the ratios of the [O III] $52 \mu \mathrm{m}$ and $88 \mu \mathrm{m}$ lines. It was adjusted until the average of the observed ratio equaled the average of the computed ratio for the 6 map positions. Figure 4 shows the ionization structure of the spherically symmetric model with $\mathrm{T}_{\text {eff }}=39,000 \mathrm{~K}$. The effect of the high density bar at $2^{\prime}$ on the Ionization is striking. Moreover, the $\mathrm{N}^{++}$zone is bigger than the $\mathrm{O}^{++}$zone, as expected. We note that the fraction of $\mathrm{N}^{+++}$is very small.

Figure 5 compares observed optical and infrared line intensities and ratios plotted against distance from the Trapezium with model predictions. The optical Iine ratios were chosen because they are not as sensitive as the individual line Intensities to errors in the electron density structure deduced from the 
deconvolution of the radio maps. We make detailed comparison of $\mathrm{O}^{+}$and $\mathrm{O}^{++}$ lines because they characterize the ionization structure. We are also interested in the ionization structure of nitrogen. $\mathrm{Ne}^{++}$is important because its $41 \mathrm{eV}$ Ionization potential makes it extremely sensitive to the effective temperature of the exciting star. We do not discuss sulfur and argon in this paper because we do not have good observational data for all the important ionization states. The [S IV] line at $10.5 \mu \mathrm{m}$ has been a notable problem in matching theory with observations (RHE and SR) and there are no observations with a sufficiently large chopper throw. [Ar II] measurements for the orion Nebula have not been published. Since there are no approprlate hydrogen lines to ratio to the infrared lines, the $36 \mu \mathrm{m}$ [Ne III] and the $52 \mu \mathrm{m}$ [O III] Iine intensities are plotted directiy; like the radio continuum, they are direct functions of the luminosity of the exciting star as well as its effective temperature.

These spherically symetric one-component models give a reasonably good account of the doubly ionized species (Figures $5 a, b, c, d$, and e). However, they do not describe well the singly ionized species (Figures $5 \mathrm{f}$ and $\mathrm{g}$ ) at positions near the center. The observed high intensities imply many more singly ionized atoms throughout the $H$ II region than predicted, but particularly in the central 2'. Only a model with $\mathrm{T}_{\text {eff }} \leq 37,000 \mathrm{~K}$ could conceivably fit the [0 II] and [N II] data. In addition, the density implied by the singly ionized [O II] $3729 / 3726$ ratio (Figure $5 \mathrm{~h}$ ) of Osterbrock and Flather (1959) and Caplan (1972) implies a density roughly 1.5 times larger than that implied by the model ratio. Since most of the $0^{+}$is in the low density outer parts, the clumping would have to be much larger in the $0^{+}$zone than is required for $0^{++}$in order to produce the observed $3729 / 3726$ ratio. Figures $51-0$ will be discussed in the next 
section, where more detailed comparisons with the observations are made.

\section{b) Bar/Halo Models}

Some of the shortcomings of the one component models are probably due to their geometric simplicity; for example, Zuckerman (1973) suggested that the Orion Nebula should not be treated as spherically symmetric. As an alternative to spherically symmetric models, we derived a two component bar/halo model of the Orion Nebula which we will compare to the standard one component models discussed above. The two component model consists of two hemispheres, one in front of the star along the line of sight (also called the "halo" model) and one in back (the "bar" model) where the erosion of the molecular cloud is occurring. Each hemisphere is taken as half of a spherically symmetric model in the computation. Figure 6 is a schematic drawing of the bar/halo models. We choose this geometry as a first attempt at non-sphericity because the bar as seen in optical photographs is clearly an lonization front (see Goudis 1982 for a review) but yet there is low density ionized material extending to great distances from the Trapezium. The electron densities are given in Fig. 3 . The clumping factor derived from the $52 / 88 \mu \mathrm{m}$ ratio for the bar/halo models is 3.0 . The front hemisphere has a high density at the center, with density decreasing monotonically out to the edge. The back hemisphere starts with the same high central density, decreases sharply, but then increases again to an ionization front at $0.29 \mathrm{pc}\left(2^{\prime}\right.$ at $\left.500 \mathrm{pc}\right)$ from the star. This reproduces the bar in projection. The bar/halo geometry reproduces the same radio flux as the one component models. The chief advantage of the bar/halo models is that the bar hemisphere has its singly lonized lons at high densities concentrated with in the central 2'. The bar/halo models are also intended to reproduce only the 
observations along our cut south from the Trapezium.

Figure 7 shows the predictions of the bar/halo models and the observations. (The sharp discontinuities at $2^{\prime}$ are due to the edge of the bar.) The bar/halo models fit the data for singly ionized species significantly better than the one component models. However, it is apparent that even this departure from spherical symmetry is not sufficient to explain all aspects of the Orion Nebula; more realistic models (which are beyond the scope of this paper) are still needed. On the other hand, for doubly ionized species the two types of models give very similar answers. Although geometry can certainly explain some of the discrepancies of the predicted intensities with the observations, particularly the predicted electron density sensitive lines, we show below that other discrepancles must have other causes.

\section{DETAILED COMPARISON OF OBSERVATIONS AND MODELS}

\section{a) Oxygen}

Our data, plotted in Figs. $5 a$ and $7 \mathrm{a}$, show that the bar region is less dense than the trough region, because the $52 / 88 \mu \mathrm{m}$ ratio decreases monotonically with distance from the Trapezium. The bar is less dense in [0 II] as well

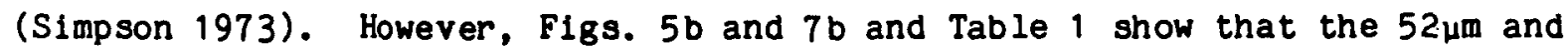
$88 \mu \mathrm{m}$ intensities increase at the bar, imply ing a longer pathlength through the bar than through the trough, or alternatively, a smaller clumping factor in the bar. The clumping factor represents a way of accounting for inhomogeneities in models that are unrealistically simple by computational necesity. Our models assume that the clumping factor is independent of position. Moreover, because of the requirements of spherical symmetry, there is very dense bar material in the models at all lines of sight out to $2^{\prime}$ (F1g. 6 ), requiring that the material 
In the trough line of sight be essentially zero. The lack of agreement between the observations and the model predictions in Figs. 5a and 7a shows that spherically symmetric geometries or even our bar/halo geometry are not appropriate for the Orion Nebula. However, one can partially compensate for the Inadequate geometry of the models in abundance analyses by averaging nearby points.

The predicted $3729 / 3726$ ratio in the bar/halo models (F1gs. $7 \mathrm{~h}$ ) now implies too high a density; this is undoubtably the same geometry effect discussed above (1.e. the bar is too dense).

We next compare our infrared data and the optical data of PTP, TPPD, and LDR through the ratio [O III] $52 \mu \mathrm{m} / 5007 \mathrm{~A}$ (Figs. 51 and 71 ). This ratio was constructed from data points that overlapped spatially, albeit imperfectly. The ratio is sensitive to electron density $\mathrm{N}_{\mathrm{e}}$ through the $52 \mu \mathrm{m}$ line and to electron temperature $T_{e}$ through the 5007A line. (Higher density decreases the density. sensitive line intensities through collisional de-excitation and hlgher electron temperature increases the intensities of the temperature sensitive forbldden Iines.) The models show good agreement with the measured line ratios in the core, but the model ratios are too high in the halo. This implles that either $T_{e}$ or $\mathrm{N}_{\mathrm{e}}$ is too low in the models. The latter is not likely because the bar/halo models have higher $\mathrm{N}_{e}$ (Fig. 3) and the agreement is worse. In fact, the predicted $52 / 88 \mu \mathrm{m}$ ratio in the bar/halo models (Fig. $7 \mathrm{a}$ ) shows that $\mathrm{N}_{e}$ is too high in the halo compared to our observations. The optical ratio $5007 / 4363 \mathrm{~A}$ (Figs. $5 j$ and $7 j$ ) shows that $T_{e}$ is too low in all but perhaps the very centers of the models. (The 4363A line is more sensitive to electron temperature than the 5007A line because it comes from a higher energy level). The bar/halo 
models are less discrepant in the cores than the one component models. Most of the discrepancy is probably due to [O III] 4363A (a favorite electron temperature indicator), because the $52 / 5007$ and $5007 / \mathrm{HB}_{\beta}$ rat10s, and the 108 of

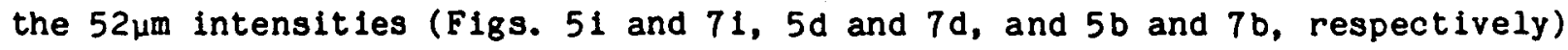
all agree better with the observations than does the $5007 / 4363$ ratio. If the problem is the electron temperature in the models (rather than the cross section for the $4363 \mathrm{~A}$ line), $T_{e}$ would have to be raised from $-7000 \mathrm{~K}$ at $2.5^{\prime}$ from the center to over $8000 \mathrm{~K}$ to provide the required agreement (but see the later section on neon).

The optical ratio [O II] $(3726+3729) / H \beta$ (F18s. $5 f$ and $7 f$ ) shows that there is not enough singly ionized oxygen, particularly in the one component model. The bar/halo model gives a better fit but not in the central 1'. The inadequacy of the model predictions cannot entirely be attributed to abundance, because

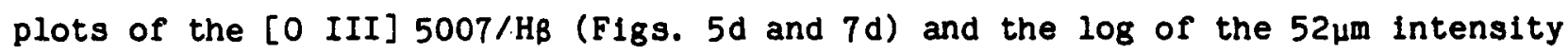
(Figs. 5b and 7b) show better agreement with the observations. Some of the excess [0 II] $3727 \AA$ observed in the center may be due to scattering; however, this must be a small effect because the absolute flux at $3727 \mathrm{~A}$ is so much larger in the center than at 4 ' from the center. A more likely explanation is that the assumed stellar atmospheres are inappropriate. An exciting star with $\mathrm{T}_{\text {eff }}<37,000 \mathrm{~K}$ seems to be necessary for agreement with $0^{+}$, although $0^{++}$agreement is better with $\mathrm{T}_{\text {eff }}=37-38,000 \mathrm{~K}$. If the exciting star in the models were less luminous (1.e. had a smaller radius), the $0^{++}$Stromgren spheres would be smaller (too small for the radio maps) and the strong decrease in [0 III] intensities seen in Figs. 5b, 5d, 7b, and $7 \mathrm{~d}$ that marks the Stromgren sphere would come at smaller distances from the Trapezium. This would help the $\mathrm{O}^{++}$agreement, but not the $0^{+}$ agreement. 


\section{b) Nitrogen}

As with singly fonized oxygen, the models need more singly ionized nitrogen, as can be seen in the $6584 / \mathrm{HB}$ ratio in Figs. $5 \mathrm{~g}$ and $7 \mathrm{~g}$ when the stellar effective temperature is $>37,000 \mathrm{~K}$. Although scattering should not contribute as much to the intensity of the $6584 \AA$ line as to that of the $3727 \AA$ Iine (because the cross section Increases as $1 / \lambda$ ), the $6584 / \mathrm{HB}$ excess appears to be as large as the $(3726+3729) / H \beta$ excess (Figs. $5 f$ and $7 f$ ). The electron temperature, as determined from the $6584 / 5755$ ratio (F1gs. $5 \mathrm{k}$ and $7 \mathrm{k}$ ), could be higher for the one component model, but is satisfactory for the bar/halo models, particularly in the core. Even if the electron temperatures of the models were increased within reasonable limits, thereby increasing the [N II] $6584 / \mathrm{HB}$ ratio (Figs. $5 \mathrm{~g}$ and $7 \mathrm{~g}$ ), the models would still require more $\mathrm{N}^{+}$. Since the input $\mathrm{N} / 0$ ratio $(0.1125)$ is derived from $\mathrm{N}^{+} / 0^{+}$, the agreement in Figs. $5 l$ and $7 \ell$ is expected to be good; in fact the ratio could be $15 \%$ larger in the bar/halo model, and $35 \% \mathrm{higher}$ in the one component model. The N/O ratio for the sun is 0.1175 (Aller 1984).

On the other hand, the observed $57 / 52 \mu \mathrm{m}$ ratio (Figs. $5 \mathrm{~m}$ and $7 \mathrm{~m}$ ) of $\mathrm{N}^{++} / 0^{++}$ is about a factor of 2 larger than any model ratio in spite of the fact that the $\mathrm{N}^{++}$zone is blgger than the $\mathrm{O}^{++}$zone for all the models. Likewise, the derived $\mathrm{N}^{++} / 0^{++}$ratio is larger than the optical $\mathrm{N}^{+} / 0^{+}$ratio, a problem common to other H II regions (Lester et al. 1983, Dinerstein et al. 1984). Previous proposed solutions for this discrepancy included the effects on the fonization equilibrium of having a low ef fective temperature for the exciting star (Rubin 1983, Dinerstein et al. 1984), a large amount of collisional de-excitation in the dense gas near the star where the lonization levels are similar (this gives 
more weight to the low density material far from the star where nitrogen is more Ionized than oxygen), or optlcal depth effects in the infrared lines (Lester et al. 1983). The models show that the differences in the $\mathrm{N}^{++}$and $\mathrm{O}^{++}$sampled at various lines of sight appear only at large distances (impact parameters) from the center ( $>2^{\prime}$ to $\left.3^{\prime}\right)$. This eliminates the first two proposed solutions. Moreover, the agreement of the $52 \mu \mathrm{m}$ fluxes with the models (Figs. $5 \mathrm{~b}$ and $7 \mathrm{~b}$ ) and with the 5007A line (Figs. 51 and 71 ) shows that the optical depth at $52 \mu \mathrm{m}$ (and $88 \mu \mathrm{m})$ is small. We clearly see the advantage of mapping the nebula, because we can immediately eliminate all explanations due to ionization equilibrium!

Surviving potential solutions to this problem include errors in the electron density or in the atomic data. Since [O III] $52 \mu \mathrm{m}$ and [N III] $57 \mu \mathrm{m}$ have almost the same sensitivity to electron density when the current cross sections are used, errors in the assumed electron densities are probably not the solution. If the cross sections are in error, more than one cross section must be wrong. If just the [N III] $57 \mu \mathrm{m}$ cross section were too low, raising it by a factor of two would change the intensity by only a small amount because the densities in the models are already so high that the 57um line is collisionally de-excited to a large extent. If the cross section for the [0 III] $52 \mu \mathrm{m}$ line were decreased by a factor of two, the $5007 \mathrm{~A}$ cross section would also have to be correspondingly decreased because the $52 \mu \mathrm{m} / 5007 \mathrm{~A}$ ratio agrees with the observations (FIg. 71). The agreement with the $5007 / 4363$ ratio (Fig. $7 \mathrm{j}$ ) would then be improved and the derived oxygen abundance would be closer to that of the sun $\left(\mathrm{O} / \mathrm{H}=8.1 \times 10^{-4}\right.$, Aller 1984).

It seems most probable that the nitrogen abundance really should be a factor of two larger than that used in our models, in spite of the resulting disagreement 
with the optically derived $\mathrm{N}^{+} / 0^{+}$ratio (and the solar $\mathrm{N} / 0$ ratio). If the [N II] cross sections need to be revised, those for [O II] $3726 \mathrm{~A}$ and $3729 \mathrm{~A}$ would have to be increased as well because of the apparent agreement shown in Figs. $5 l$ and $7 \ell$. This would give better agreement with the [O II] $3727 / \mathrm{HB}$ ratios (Figs. $5 f$ and $7 f$ ) as well as the [N II] $6584 / \mathrm{HB}$ ratios (Figs. $5 \mathrm{~g}$ and $7 \mathrm{~g}$ ).

\section{c) Neon}

The [Ne III] $3869 / \mathrm{HB}$ ratio (Figs. $5 \mathrm{e}$ and $7 \mathrm{e}$ ) and the plot of the log of the $36 \mu \mathrm{m}$ intensity (Figs. $5 \mathrm{c}$ and $7 \mathrm{c}$ ) both require $\mathrm{T}_{\text {eff }}=39-40,000 \mathrm{~K}$. One could say that the [O III] $52 \mu \mathrm{m}$ Iine could fit a higher temperature star too if only the inner 4 data points (to $3^{\prime}$ ) were considered. However, there is little difference between the 5 models for the $52 \mu \mathrm{m}$ line in this region, and only the lower $\mathrm{T}_{\text {eff }}$ is consistent with the outer data points. On the other hand, there is much greater discrimination between the varlous models in the inner $3^{\prime}$ region

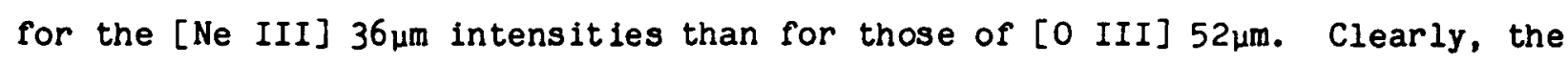

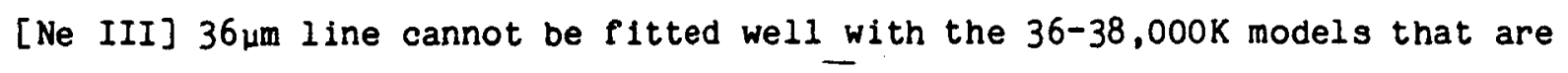
required for oxygen and nitrogen. The optical [Ne III] and [O III] observations give the same results as the infrared, thus showing that the problem is the Ionization equilibrium (or stellar atmosphere), and probably not the collisional excitation cross sections. However, the latter may be responsible for the disagreement in the electron temperatures derived from each element (the oxygen and nitrogen 1 ines showed that the electron temperatures are too low in the models, but the [Ne III] $36 \mu \mathrm{m} / 3869 \AA$ ratio (Figs. $5 \mathrm{n}$ and $7 \mathrm{n}$ ) shows that the electron temperature is too high in the models).

Unlike nitrogen and oxygen which are mostly doubly ionized, most of the 
neon in the models is in the $\mathrm{Ne}^{+}$ionization state. The only forbidden line of $\mathrm{Ne}^{+}$ is at $12.8 \mu \mathrm{m}$, where most of the ground based telescopes chop with a throw smaller than the nebula. The [Ne II] observation with the largest chopper throw (100") is that of Lester (1982; pers.comm). He measured fluxes of $8-12 \times 10^{-18} \mathrm{~W} \mathrm{~cm}^{-2}$ in 5 positions on a grid Just west of $\theta^{1}$ Or $1 \mathrm{C}$ with a $10^{\prime \prime}$ beam. His [Ne II] fluxes are plotted in Figs. 50 and 70 . The $\mathrm{Ne}^{+}$intensities are well fitted by models with $\mathrm{T}_{\text {eff }}=37-38,000 \mathrm{~K}$. As with $\mathrm{N}^{+}$and $\mathrm{O}^{+}$, there is too much singly lonized neon for the higher $T_{\text {eff }}$ models implied by the doubly lonized specles. For neon, a compromise with $\mathrm{T}_{\text {eff }}-39,000 \mathrm{~K}$ and the $\mathrm{Ne} / \mathrm{H}$ abundance higher by 20-50\% fits both Ionization states reasonably well. However, this $T_{\text {eff }}$ does not agree with the oxygen and nitrogen data. A better fit might be obtained if the emergent stellar flux resembled that of the Kurucz $37,000 \mathrm{~K}, \log \mathrm{g}=4.0$ model (Kurucz 1981) from $13.6 \mathrm{eV}$ to $41 \mathrm{eV}$, but with the flux decreasing less rapidly from $41 \mathrm{eV}$ to $54 \mathrm{eV}$.

\section{v. SUMMARY}

We present new observations of the infrared lines of [N III] $57 \mu \mathrm{m}$ and [O III] $52 \mu \mathrm{m}$ and $88 \mu \mathrm{m}$ at 6 positions and [Ne III] $36 \mu \mathrm{m}$ at 4 positions in a line south from the Trapezium in the Orion Nebula. From the data we derive electron densities and $\mathrm{N}^{++} / 0^{++}$ratios at all 6 positions.

We also present new models of the Orion Nebula, both spherically symmetric and two component bar/halo models, in which the electron density distributions are constrained to reproduce the high resolution radio surface brightnesses at the same positions as our data. The stellar effective temperatures range from $37,000 \mathrm{~K}$ to $40,000 \mathrm{~K}$ with $108 \mathrm{~g}=4.0$ or 4.5 . The stellar Lyman cont inuum fluxes are $10^{49}$ photons $\mathrm{s}^{-1}$. The two-component bar/halo models give a somewhat better 
account of the singly lonized atoms.

We compare the predicted intensities from the models to infrared and optical data to investigate a number of issues:

\section{a) Elemental Abundances}

The abundance ratio $\mathrm{O} / \mathrm{H}=4.0 \times 10^{-4}$ used in the models, which is approximately half solar, agrees satisfactorily with the observations. However, given the current cross sections, there is enough discrepancy between the $\mathrm{O}^{+}$and the $\mathrm{O}^{++}$ ionization structure that the uncertainty is large. The $\mathrm{Ne} / \mathrm{H}$ abundance ratio is almost certainly larger than the $8.1 \times 10^{-5}$ used in the models; $1.3 \times 10^{-4}$ is probably a better value, although then the Ne/O ratio would be larger than the ratio found in the sun and most planetary nebulae (Aller and Czyzak 1983, Aller 1984). Based on our [N III] and [O III] measurements we derive a N/O ratio of 0.21. The ratio from the optical [N II] and [O II] Iines is $N / O=0.11$. [N III] and [O III] should be a better indicator of $N / O$ because $\theta^{2}$ ori $C$ is hot enough that nitrogen and oxygen are mostly doubly ionized in the nebula. Because of this, we revise the nitrogen abundance upward to $\mathrm{N} / \mathrm{H}=8.4 \times 10^{-5}$. Thus we find that nitrogen and neon are approximately solar in abundance, but compared to the sun, oxygen is underabundant. It is conceivable that the missing oxygen is tied up in dust grains, although this hypothesis has previously been rejected for the Orion Nebula (e.g. Peimbert 1982, Mathis 1985).

\section{b) Stellar Effective Temperature}

The [O III] and [N III] Iines imply that the effective temperature of the exciting star is cooler than $37,000 \mathrm{~K}$. However, the [Ne III] 11nes, which are more sensitive to $\mathrm{T}_{\text {eff }}$, lead us to conclude that the effective temperature is 
$\geq 39,000 \mathrm{~K}$ for elther model geometry. With no changes in abundances, the observed [Ne II], [N II], and [O II] line fluxes would require that $\mathrm{T}_{\mathrm{eff}}$ be $37,000 \mathrm{~K}$, $<37,000 \mathrm{~K}$, and $<37,000 \mathrm{~K}$, respectively. However, with the higher abundances for neon and nitrogen discussed in the previous section, the singly and doubly Ionized species can both be fitted by temperatures of $37,000 \mathrm{~K}$ for nitrogen and 39,000K for neon. Oxygen cannot be fitted easily, but certainly implies $T_{\text {eff }} \leq 37,000 \mathrm{~K}$. Thus in this relatively unobscured $H$ II region, the $T_{\text {eff }}$ derived from nitrogen and oxygen is significantly lower than the $T_{\text {eff }}$ derived from the neon ionization. As mentioned in the introduction, in obscured H II regions the stellar effective temperature derived from the sulfur and argon ionization equilibrium is much lower than that estimated from the radio luminosity. From our additional data on neon and our mapping of the Orion Nebula in oxygen and nitrogen, we conclude that the presence of dust does not account for the inconsistencies in the methods of derivation of stellar effective temperatures. In the future, we plan to investigate other stellar spectra in the manner of RHE, such as that of a $37,000 \mathrm{~K}$ star but with additional flux beyond $41 \mathrm{eV}$ to produce additional $\sqrt{e}^{++}$. In this way, we hope to find a model that better fits the observed spectrum of the Orion Nebula.

\section{c) Scattering by Dust}

All the models show that the ratio of the singly lonized forbidden lines to $\mathrm{H} \beta$ increases faster than observed with distance from the center. If some of the visual flux observed in the center of the Orion Nebula is due to light scattered into the beam from positions far ( $\left.>2^{\prime}\right)$ from the center, there would be less need for a low $\mathrm{T}_{\text {eff }}$ to produce the large number of singly ionized atoms observed. Scattering should be much more important for [O II] 3727A than for [N II] 6584 A. 
However, the two lons show similar discrepancies, leading us to conclude that scattering is not the chlef cause of the large singly lonized line flux within $2^{\prime}$ of the center, but rather, it is the lonization equilibria and abundances. However, scattering could still affect the slope of the line/HB ratio at the center. On the other hand, our infrared oxygen and neon data show good agreement with optical data and demonstrate that scattering is also not important for doubly ionized ions in the central $4^{\prime}$ of the nebula.

\section{d) Electron Densities}

The measured [O III] $52 / 88 \mu \mathrm{m}$ line ratios show that the enhanced intensities at the ionization bar are due to geometry effects rather than increased electron densities. We therefore do not expect our present models to reproduce the details of the density-sensitive line ratios in the vicinity of the bar. An average clumping factor for the models was derived from the far infrared [O III] lines, and the density variations in the models do reproduce the general increase in the line ratios of singly lonized lons (e.g. [O II] $3729 / 3726$ ) with distance. The detailed agreement might be improved by using a different geometry/density distribution such as a blister model (Rubin 1984). We note however that the blister geometry will not account for the enhanced intensities near the bar.

\section{e) Electron Temperature}

The model electron temperatures are too high in the very center $\left(\mathrm{Ne}^{++}\right.$ zone), but low in the $\mathrm{O}^{++}$and $\mathrm{N}^{+}$zones. The $\mathrm{Ne}^{++}$and $\mathrm{O}^{++}$. zones, of course, overlap. For $\mathrm{O}^{++}$, the model temperatures are worse for [0 III] $5007 / 4363$ than for $[0$ III $] 52 \mu \mathrm{m} / 5007 \AA$. Electron temperatures are not very sensitive to $T_{\text {eff }}$ 
for the range of $\mathrm{T}_{\text {eff }}$ considered here. These discrepencies are possibly due to errors in the atomic parameters.

We thank the staff of the KAO for their continued support and assistance. We thank $P$. Duffy for $h$ is assistance with the instrument and we thank $h i m$ and J. Bregman for their careful reading of the manuscript. We also thank D. Lester for giving us $\mathrm{h}$ is data on [Ne II] in advance of publication and an anonymous referee for helpful suggestions for clarifying some points. R. H. Rubin and J. P. Simpson were supported by NASA/Ames Research Center Interchange grants NCA2-1R390-404 and NCA2-1 R690-404 respectively. 


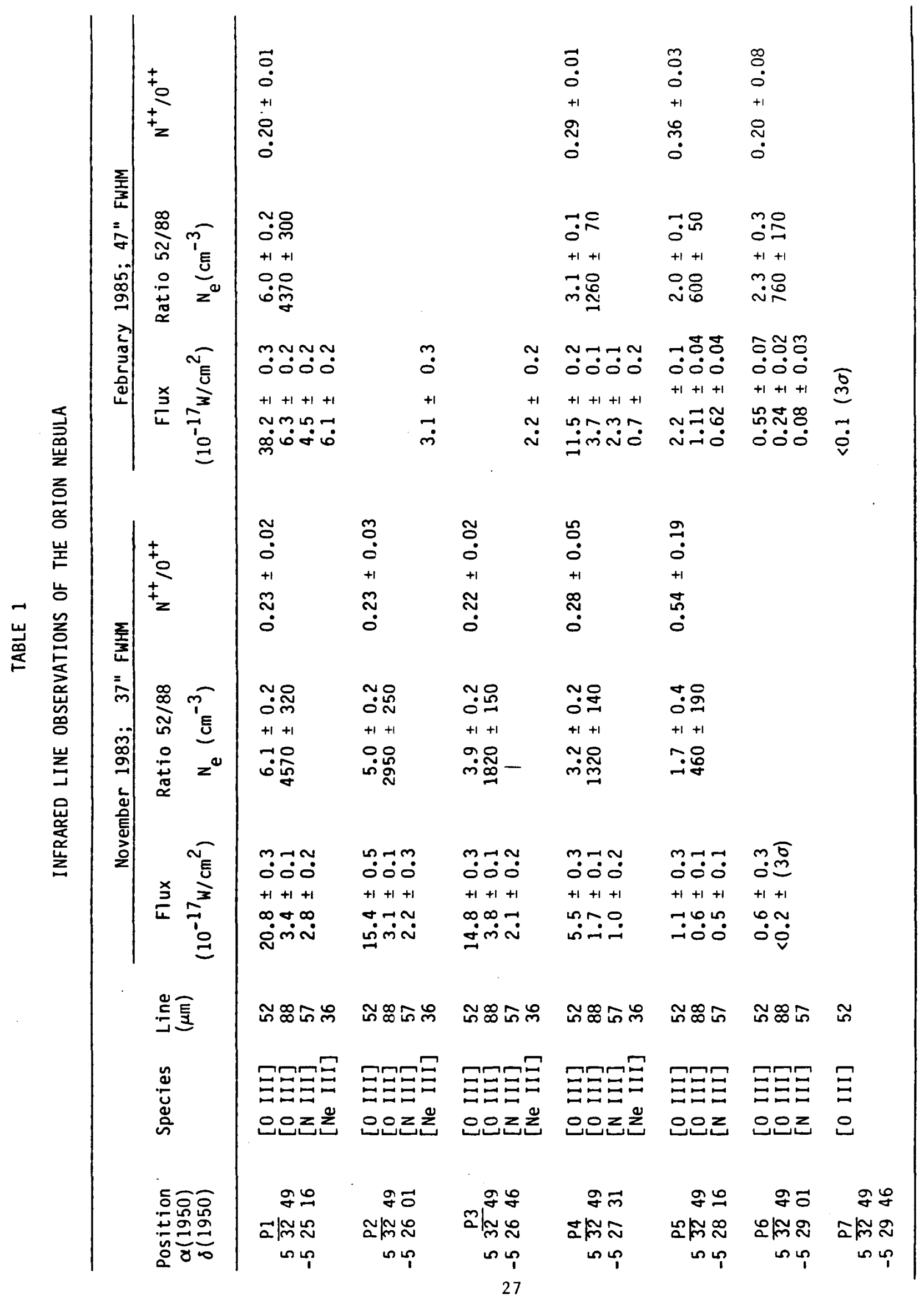


REFERE NCES

Abbott, D.C., and Hummer, D.G. 1985, Ap.J., 294, 286.

Aggarwal, K.M., Baluja, K.L., and Tully, J.A., 1982, M.N.R.A.S., 201, 923. Aller, L.H. 1984, Physics of Thermal Gaseous Nebulae (Physical Processes in Gaseous Nebulae), Astrophysics and Space Sclence Library 112 (Dordrecht: Reidel).

Aller, L.H., and Czyzak, S.J. 1983, Ap.J. Suppl., 51, 211. Bayes, F.A., Saraph, H.E., and Seaton, M.J. 1985, M.N.R.A.S., 215 , 85P. Butler, S.E., Heil, T.G., and Dalgarno, A. 1984, J.Chem. Phys., $80,4986$. Butler, K., and Mendoza, C. 1984, M.N.R.A.S., 208,17 P. Caplan, J.G. 1972, Astr.Ap., $18,404$. Cont 1, P.S. 1973, Ap.J., 179, 181 . Dinerstein, H., Lester, D., Werner, M., Watson, D., Genzel, R., and Rubin, R. 1984, NASA/A.S.P. Airborne Astronomy Symposium, NASA CP-2353, eds. H.A. Thronson and E.F. Erickson, p. 266.

Erickson, E.F., Knacke, R.F., Tokunaga, A.T., and Haas, M.R. 1981, Ap.J., 245 , 148.

Erickson, E. F., Houck, J. R., Harwit, M. O., Rank, D. M., Haas, M. R., Hollenbach, D. J., Simpson, J. P., Augason, G. C., and McKibbin, D. D. 1984a, Airborne Astronomy Symposium, NASA CP-2353, eds. H.A. Thronson and E.F. Erickson, p. 313.

Erickson, E.F., Haas, M.R., Simpson, J.P., Duffy,P., Rubin, R.H., and Houck, J.R. 1984b, Bull.A.A.S., 15, 928.

Erickson, E, F., Matthews, S., Augason, G. C., Houck, J. R., Rank, D. M., and Haas, M. R. 1985, Proceedings of the Society of Photo-Optical Instrumentation Engineers, $509,129$. 
Furniss, I., Jennings, R.E., King, K.J., Lightfoot, J.F., Emery, R.J., Nay lor, D.A., and Fitton, B. 1983, M.N.R.A.S., 202, 859.

Goudis, C. 1982, The Orion Complex: A Case Study of Interstellar Matter, Astrophysics and Space Science Library 90 (Dordrecht: Reidel).

Herter, T., Helfer, H.L., and Pipher, J.L. 1983, Astr.Ap.Suppl., $51,195$.

Johnston, K.J., and Hobbs, R.W. 1969, Ap.J., 158, 145.

Johnston, K.J., Palmer, P., Wilson, T.L., and Bieging, J.H. 1983, Ap.J., 271 , L89.

Kurucz, R.L. 1979, Ap.J. Suppl., 40 , 1.

Lacy, J.H., Beck, S.C., and Geballe, T.R. 1982, Ap.J., 255, 510.

Lee, T.A. 1968, Ap.J., 152, 913.

Lester, D.F., Dinerstein, H.L., and Rank, D.M. 1979, Ap.J., 232, 139 (LDR).

Lester, D.F., Dinerstein, H.L., Werner, M.W., Watson, D.M., and Genzel, R.L. 1983, Ap.J., $271,618$.

Martin, A.H.M., and Gull, S.F. 1976, M.N.R.A.S., 175, 235.

Mathis, J.S. 1985, Ap.J., $291,247$.

Nussbaumer, H., and Storey, P.J. 1979, Astr.Ap., 71, L5.

O'Dell, C.R., and Hubbard, W.B. 1965, Ap.J., $142,591$.

Osterbrock, D., and Flather, E. 1959, Ap.J., 129, 26.

Peimbert, M. 1982, Symposium on the Orion Nebula to Honor Henry Draper, Annals of the New York Academy of Science, 395, eds. A.E. Glassgold, P.J. Hugg ins, and E.L. Schucking, New York, p. 24 . 
Peimbert, M., and Costero, R. 1969, Bol.Obs.Ton.Tac., 5, 3.

Peimbert, M., and Torres-Peimbert, S. 1977, M.N.R.A.S., 179, 217 (PTP).

Pottasch, S.R., Wesselius, P.R., and van Duinen, R.J. 1979, Astr.Ap., 77, 189. Rodriguez, L.F., and Chaisson, E.J. 1978, Ap.J., 221, 816.

Rubin, R.H. 1968, Ap.J., 153, 761. 1983, Ap.J., 274,671 . 1984, Ap.J., $287,653$. 1985, Ap.J. Suppl., $57,349$.

Rub in, R.H., Hollenbach, D.J., and Erickson, E.F. 1983, Ap.J., 265, 39 (RHE). Schiffer, F.H.,III, and Mathis, J.S. 1974, Ap.J., 194, 597.

Schraml, J., and Mezger, P.G. 1969, Ap.J., 156, 269.

Shure, M.A., Houck, J.R., Gull, G.E., and Herter, T. 1984, Ap.J., 281, L29. Simpson, J.P. 1973, Publ.A.S.P., $85,479$. 1975, Astr.Ap., $39,43$.

Simpson, J.P., Cuzzi, J.N., Erickson, E.F., Strecker, D.W., and Tokunaga, A.T. 1981, Icarus, $48,230$.

Simpson, J.P., Haas, M.R., Rubin, R.H., and Erickson, E.F. 1984, Airborne Astronomy Symposium, NASA CP-2353, eds. H.A. Thronson and E.F. Erickson, p. 148 .

Simpson, J.P., and Rubin, R.H. 1984, Ap.J., 281, 184 (SR).

Torres-Peimbert, S., Peimbert, M., and Daltabuit, E. 1980, Ap.J., 238, 133 (TPPD).

Werner, M. W. 1982, Symposium on the Orion Nebula to Honor Henry Draper, Annals of the New York Academy of Sciences, 395 , eds. A.E. Glassgold, P.J. Huggins, and E.L. Schucking, New York, p. 79. 
Wilson, T.L., and Pauls, T. 1984, Astr.Ap., 138, 225.

Zellik, M., II 1977, Ap.J., 213, 58.

Zuckerman, B. 1973, Ap.J., 183, 863. 


\section{FIGURE CAPTIONS}

Fig. 1. The [Ne III] lines at $36 \mu \mathrm{m}$ at each of the four positions P1-P4 (top to bottom).

Fig. 2. The radio brightness temperature scaled to $5 \mathrm{GHz}$ for a cut south from the Trapezium. The HB measurements of PTP as scaled to $5 \mathrm{GHz}$ are also plotted.

Fig. 3. The electron densities in the clumps as a function of distance from the exciting star. The units of the abcissa are $0.145 p c$, which corresponds to 1 ' at a distance of 500 pc.

Fig. 4. The ionization structure in nitrogen, oxygen and neon as a function of distance from the center for the one component model with $T_{\text {eff }}=39,000 \mathrm{~K}$. The units of the abcissa are $0.145 \mathrm{pc}$, which corresponds to $1^{\prime}$ at a distance of $500 \mathrm{pc}$.

Fig. 5. Line intensities, or intensity ratios, for the five different models as functions of distance from the center of the model. The observed data are also plotted as functions of distance from the Trapezlum. With exceptions as noted, the squares are our data of November, 1983, and the triangles are our data of February, 1985. Where no error bars are plotted for our data, the error bars are smaller than the size of the plotted squares or triangles. The circles are the visible data of PTP and TPPD, and the dlamonds are the visible data of LDR. Fig. 5a. The ratio of [O III] $52 / 88 \mu \mathrm{m}$.

Fig. 5b. The log of the [0 III] $52 \mu \mathrm{m}$ intensities.

Fig. 5c. The log of the [Ne III] $36 \mu \mathrm{m}$ intensities.

F18. 5d. The ratio of [O III ] 5007A/HB. 
Fig. 5e. The ratio of [Ne III] 3869A/HB.

Fig. 5f. The ratio of [O II $](3726+3729) A / H B$.

Fig. 5g. The ratio of [N II] $6584 \mathrm{~A} / \mathrm{HB}$. The triangle is from O'Dell and Hubbard (1965).

Fig. 5h. The ratio of [O II] 3729/3726A. The circles are from Osterbrock and Flather (1959), the squares are from Simpson (1973), and the $x$ 's are from Caplan (1972).

Fig. 5i. The ratio of [0 III] $52 \mu \mathrm{m} / 5007 \mathrm{~A}$.

Fig. 5J. The ratio of [O III] 5007/4363A.

Fig. 5k. The ratio of [N II] $6584 / 5755$ A.

Fig. 5l. The ratio of [N II] 6584 A/[O II $](3726+3729) A$.

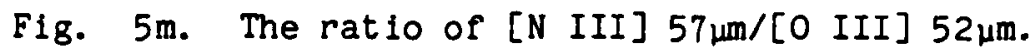

F1g. 5n. The ratio of [Ne III] $36 \mu \mathrm{m} / 3869 \mathrm{~A}$.

Fig. 50. The log of the intensity of [Ne II] $12.8 \mu \mathrm{m}$.

Fig. 6. Schematic drawing of the bar/halo models.

Fig. 7. The same as Fig. 5 for the bar/halo models. Fig. 7a. The ratio of [O III $] 52 / 88 \mu \mathrm{m}$.

Fig. 7b. The 108 of the [O III] $52 \mu \mathrm{m}$ intensities.

Fig. 7c. The log of the [Ne III] $36 \mu \mathrm{m}$ intensities.

Fig. 7d. The ratio of $[0$ III $] 5007 \mathrm{~A} / \mathrm{HB}$.

Fig. 7e. The ratio of [Ne III] $3869 \AA / \mathrm{HB}$.

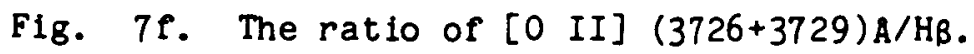

Fig. 7g. The ratio of [N II] $6584 \AA / H B$.

Fig. 7h. The ratio of [O II] $3729 / 3726$ A.

Fig. 71. The ratio of [0 III] $52 \mu \mathrm{m} / 5007 \mathrm{~A}$.

Fig. $7 \mathrm{j}$. The ratio of [0 III] $5007 / 4363 \mathrm{~A}$. 
Fig. 7k. The ratio of [N II] 6584/5755A.

Fig. 7l. The ratio of [N II] $6584 \mathrm{~A} /[\mathrm{O}$ II] $(3726+3729) \mathrm{A}$.

Fig. $7 \mathrm{~m}$. The ratio of [N III] $57 \mu \mathrm{m} /[0$ III] $52 \mu \mathrm{m}$.

Fig. 7n. The ratio of [Ne III] $36 \mu \mathrm{m} / 3869 \mathrm{~A}$.

Fig. 70. The log of the intensity of [Ne II] $12.8 \mu \mathrm{m}$. 


\section{ADDRESSES OF AUTHORS}

Edwin F. Erickson, Michael R. Haas, Robert H. Rubin, and Janet P. Simpson: Astrophysical Experiments Branch, MS-245-6, NASA/Ames Research Center, Moffett Field, CA 94025 
Fig. 1

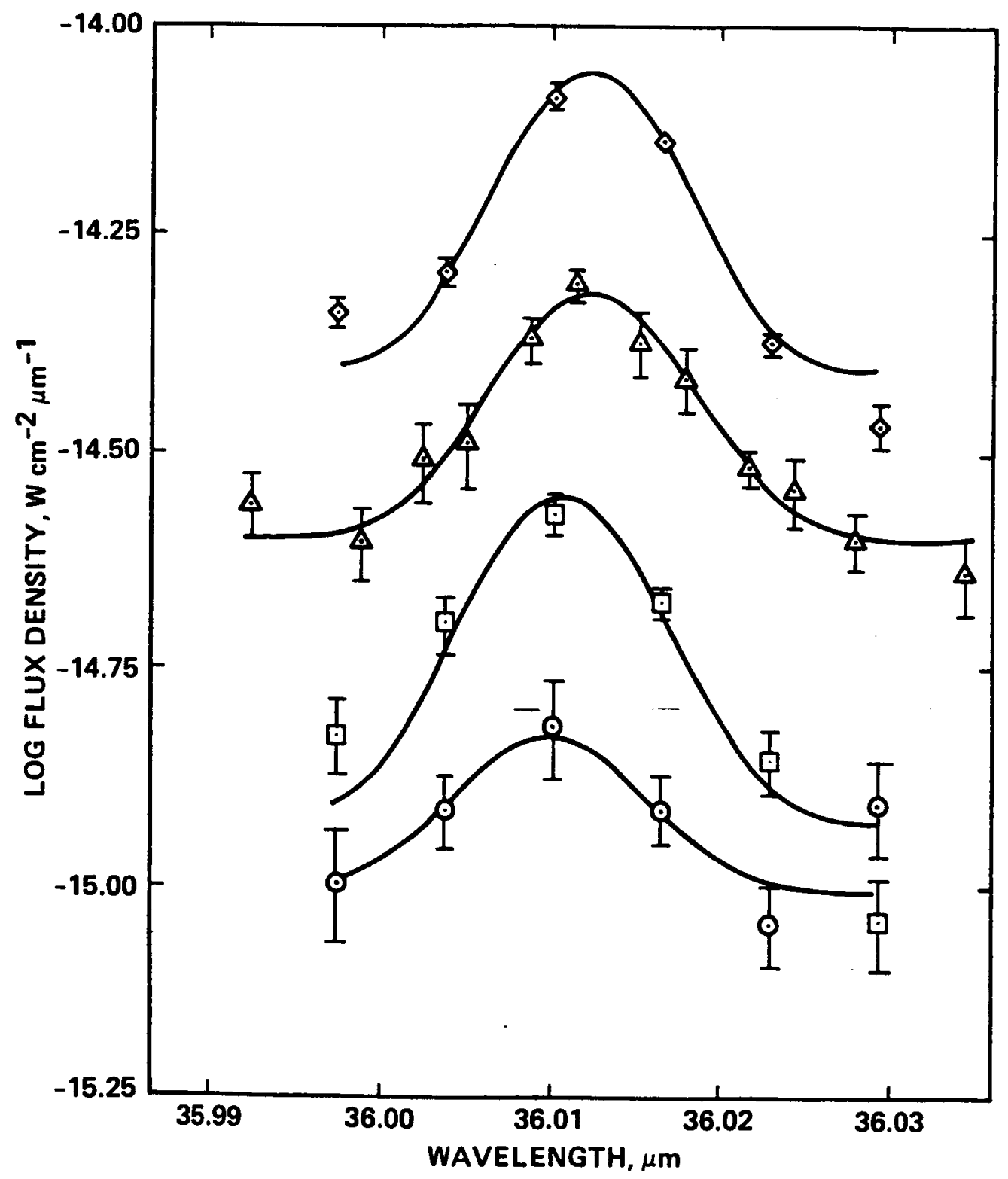

SIMPSON 
Fig. 2

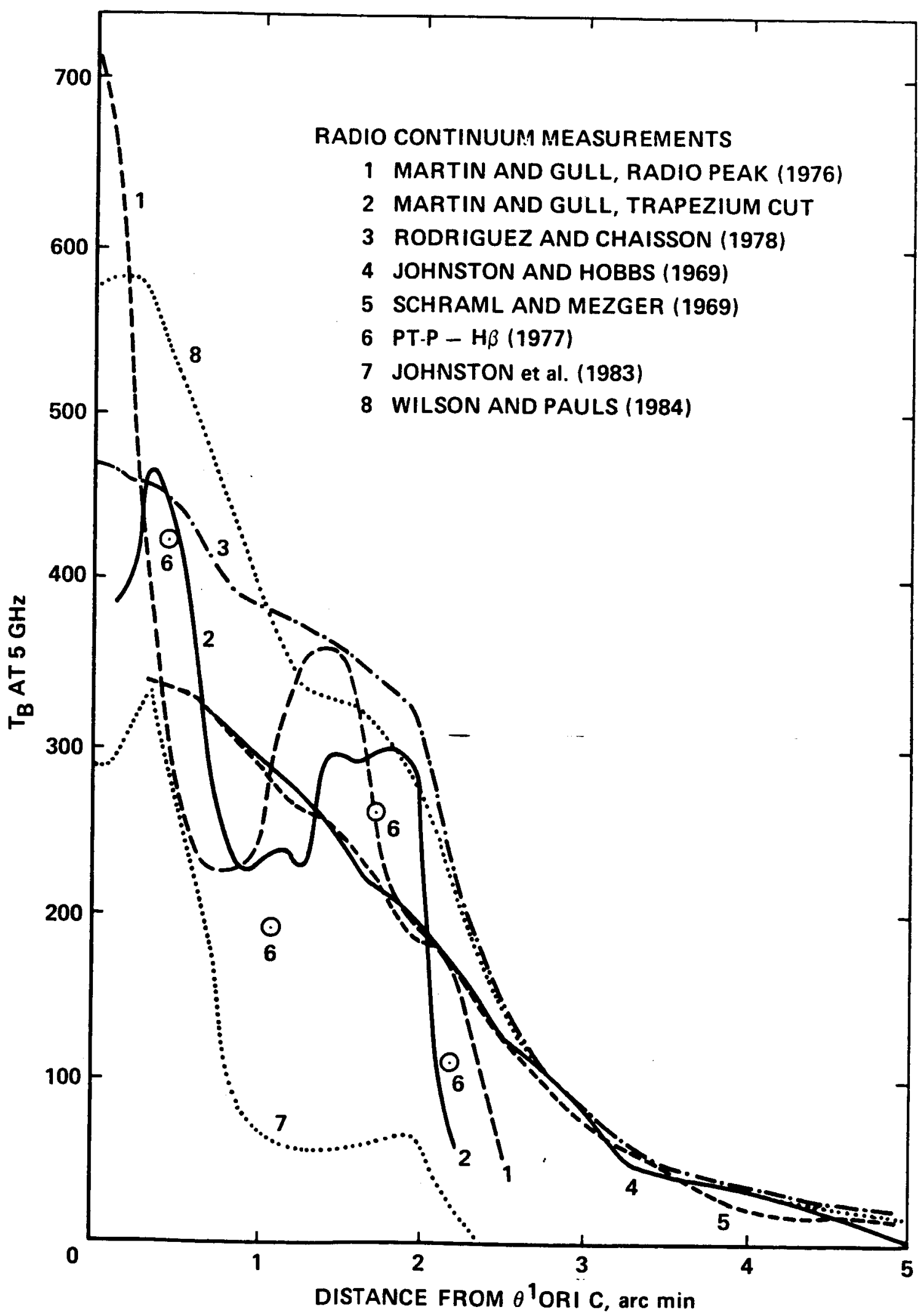


Fig 3

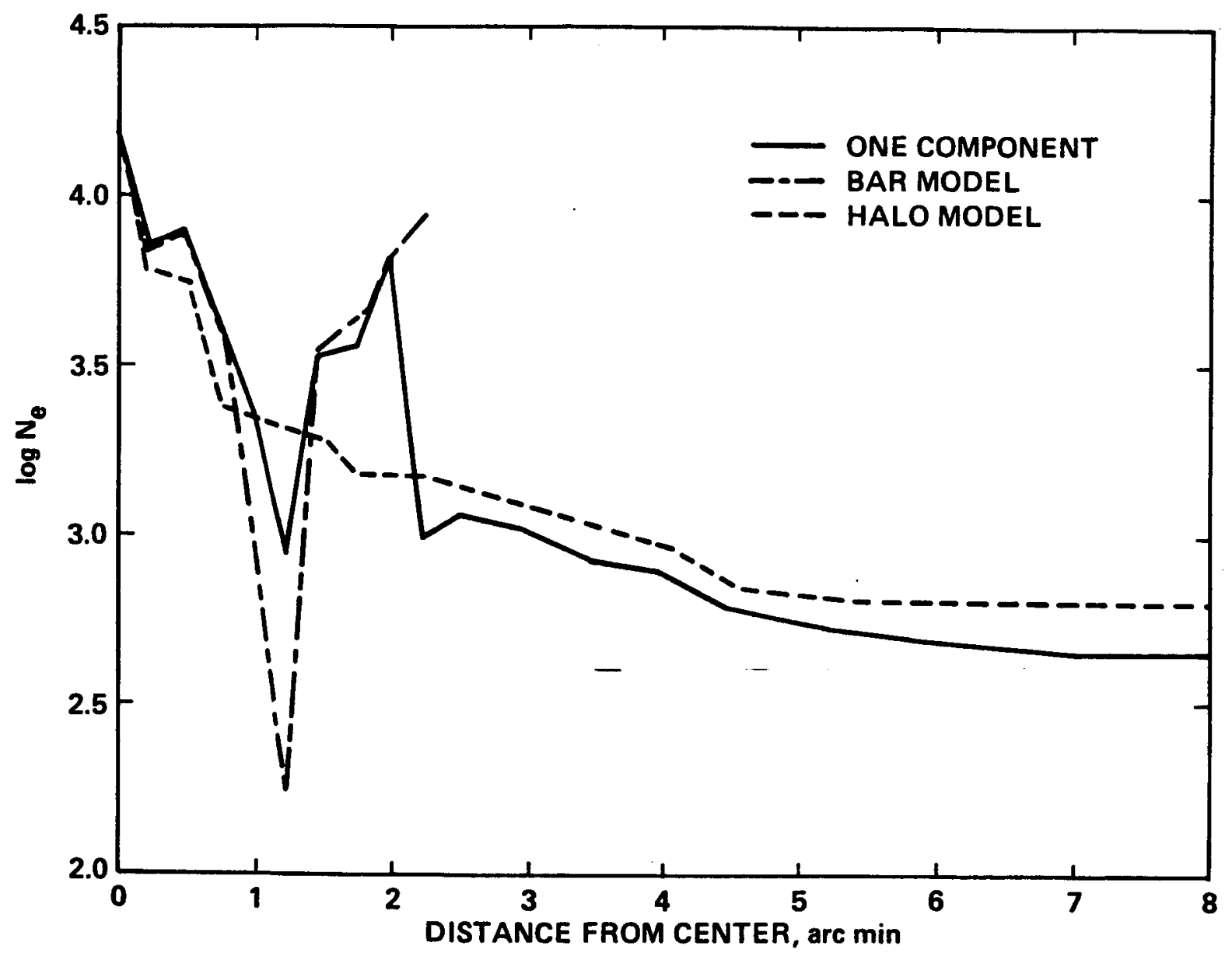

SIMPSON 
Fig 4

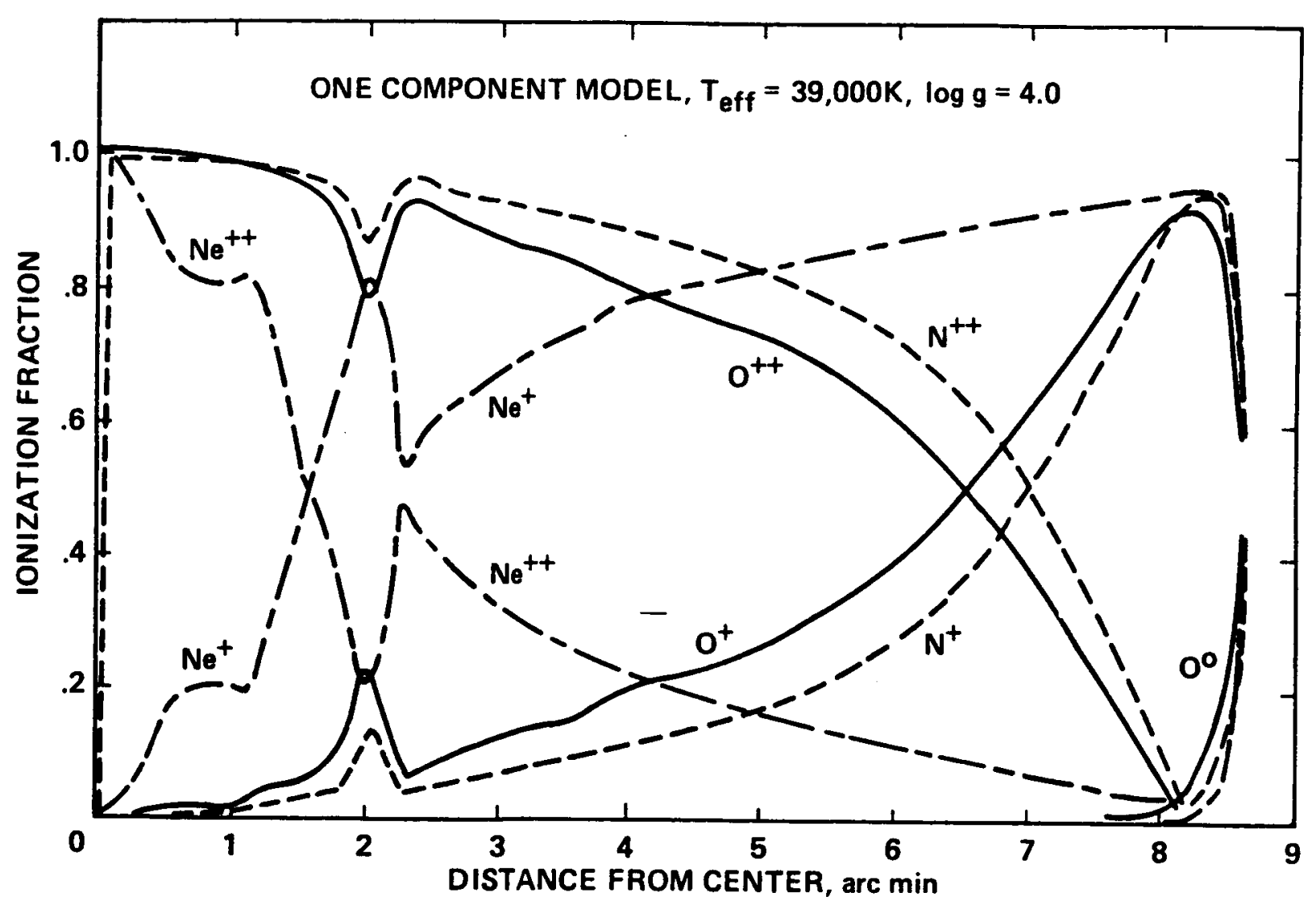

SIMPSON 

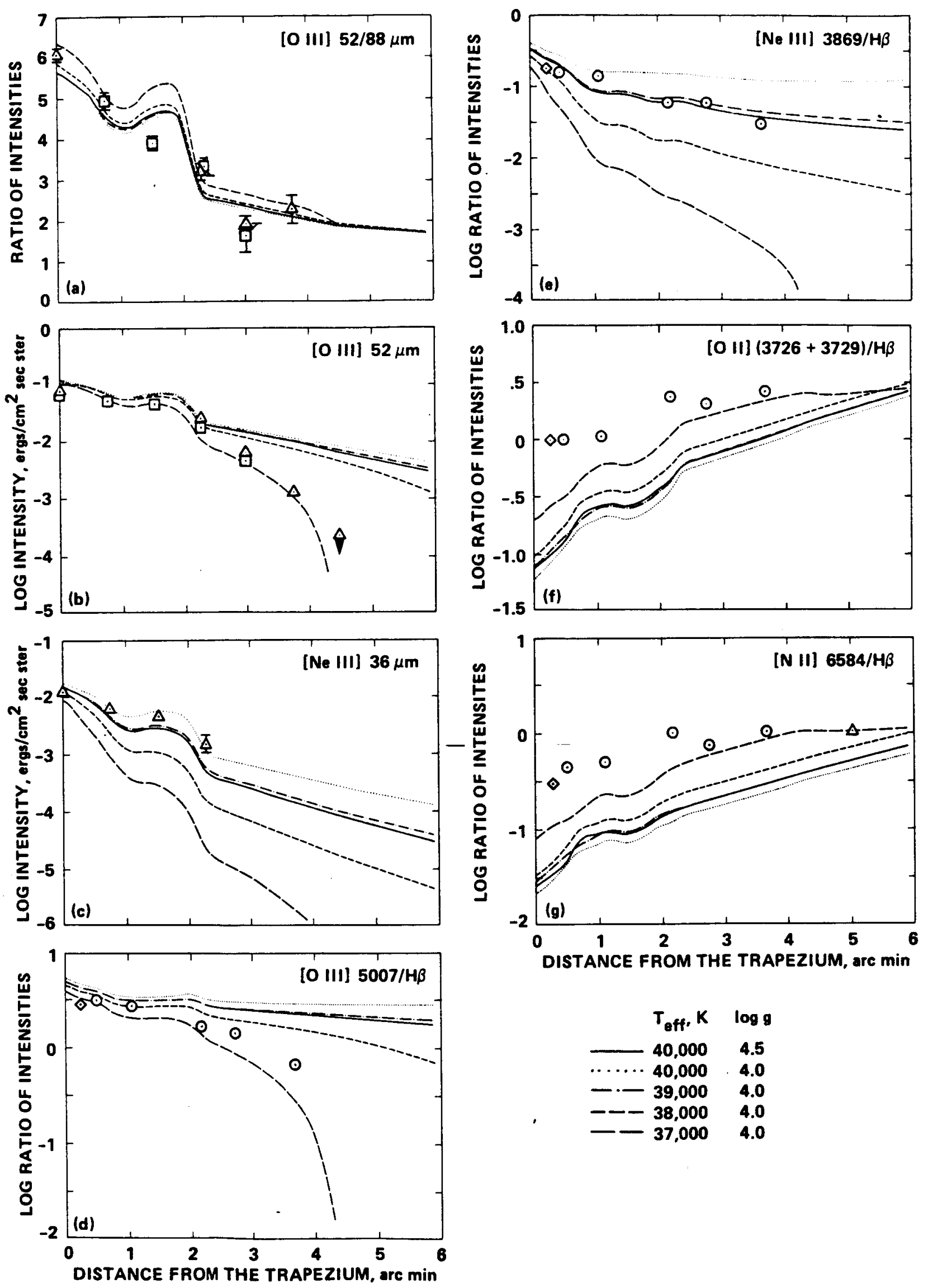

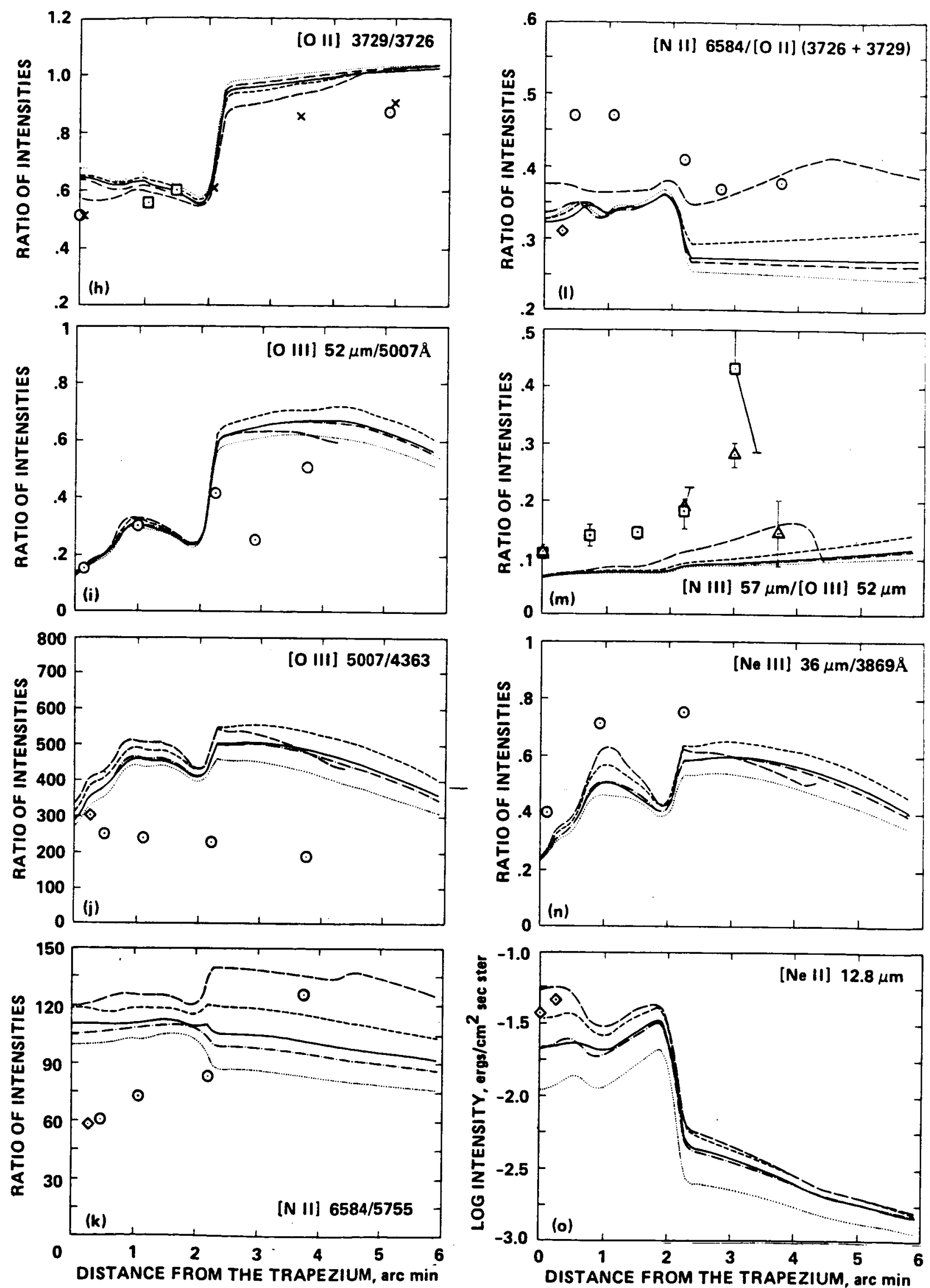


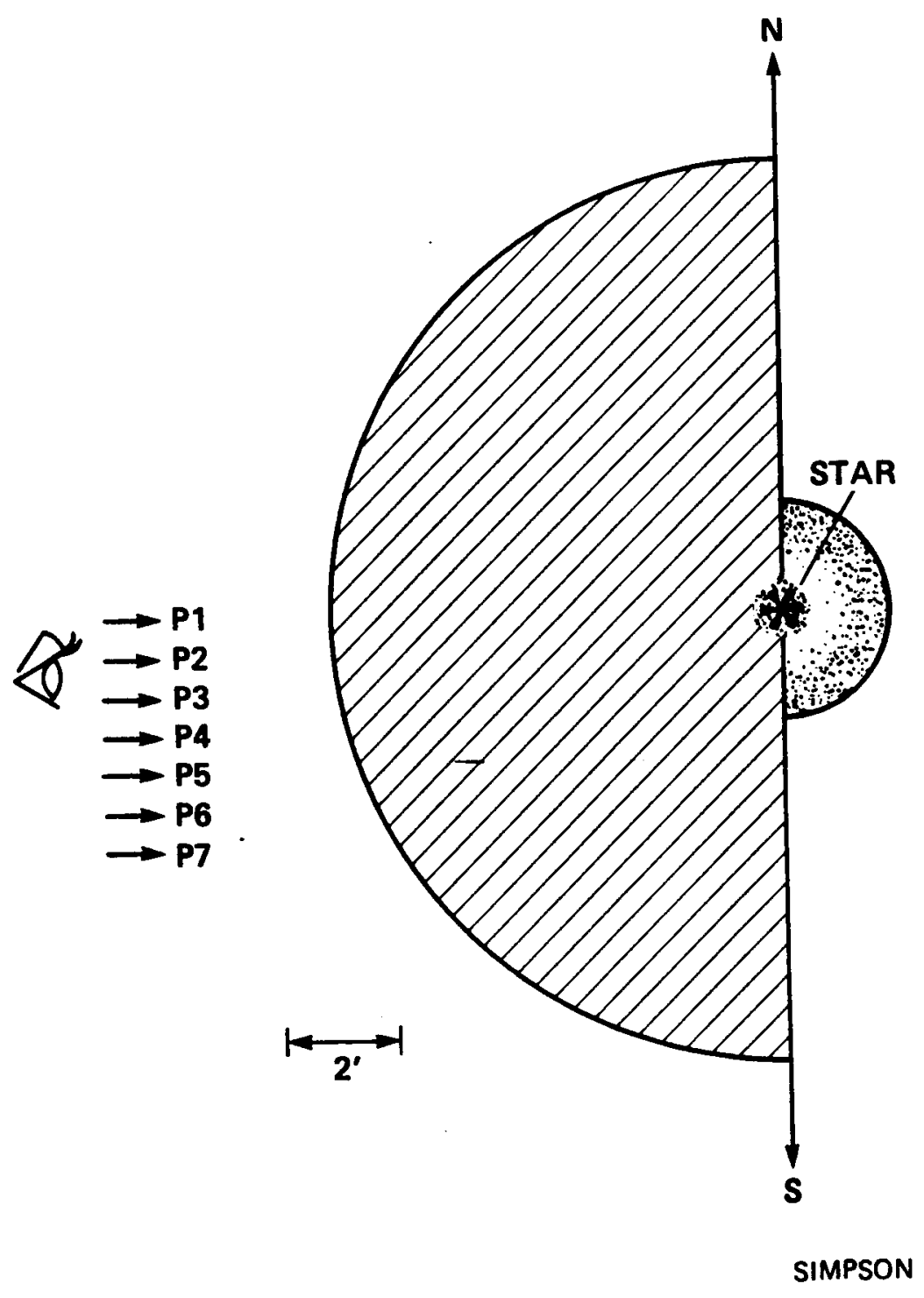



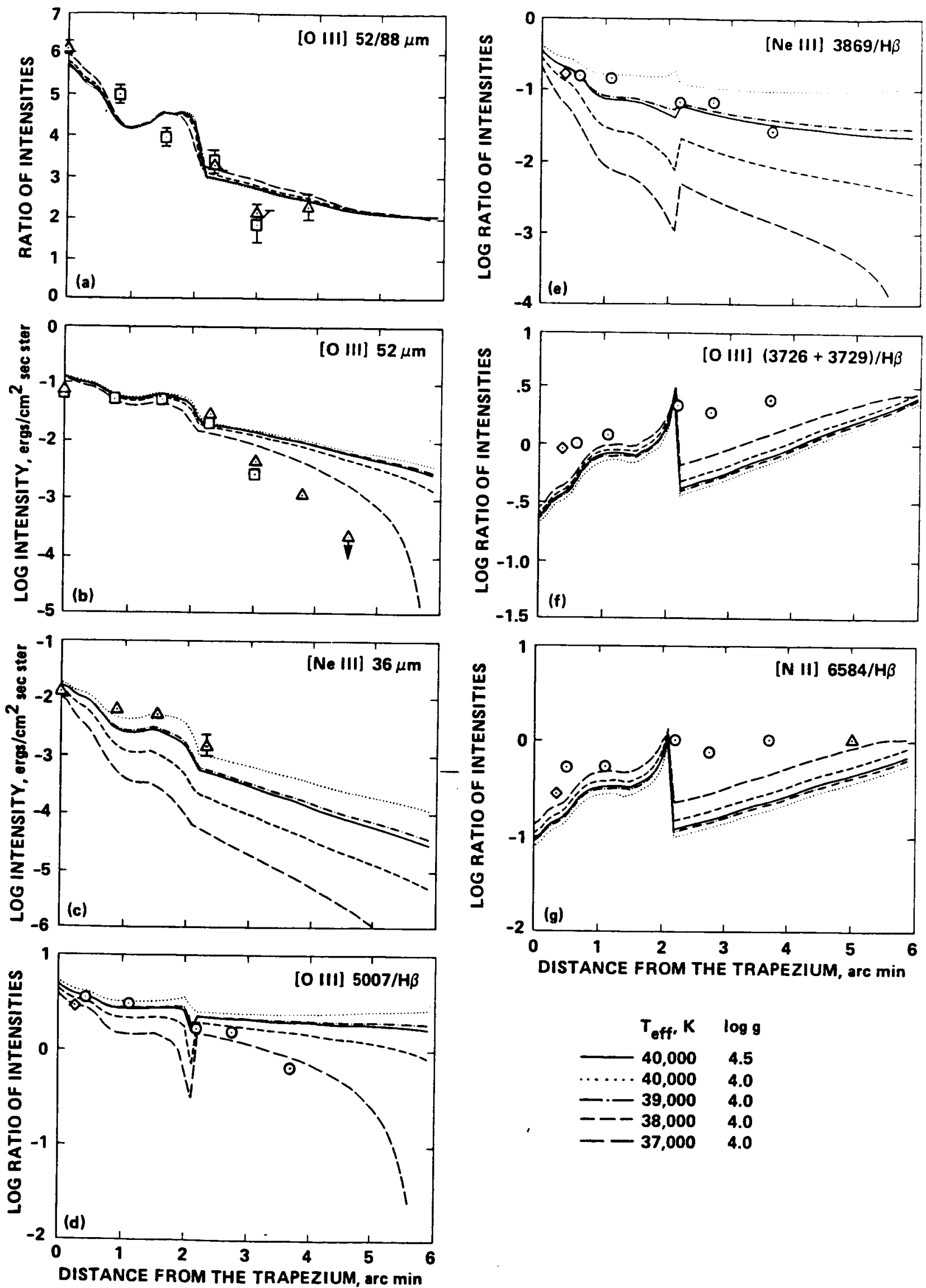

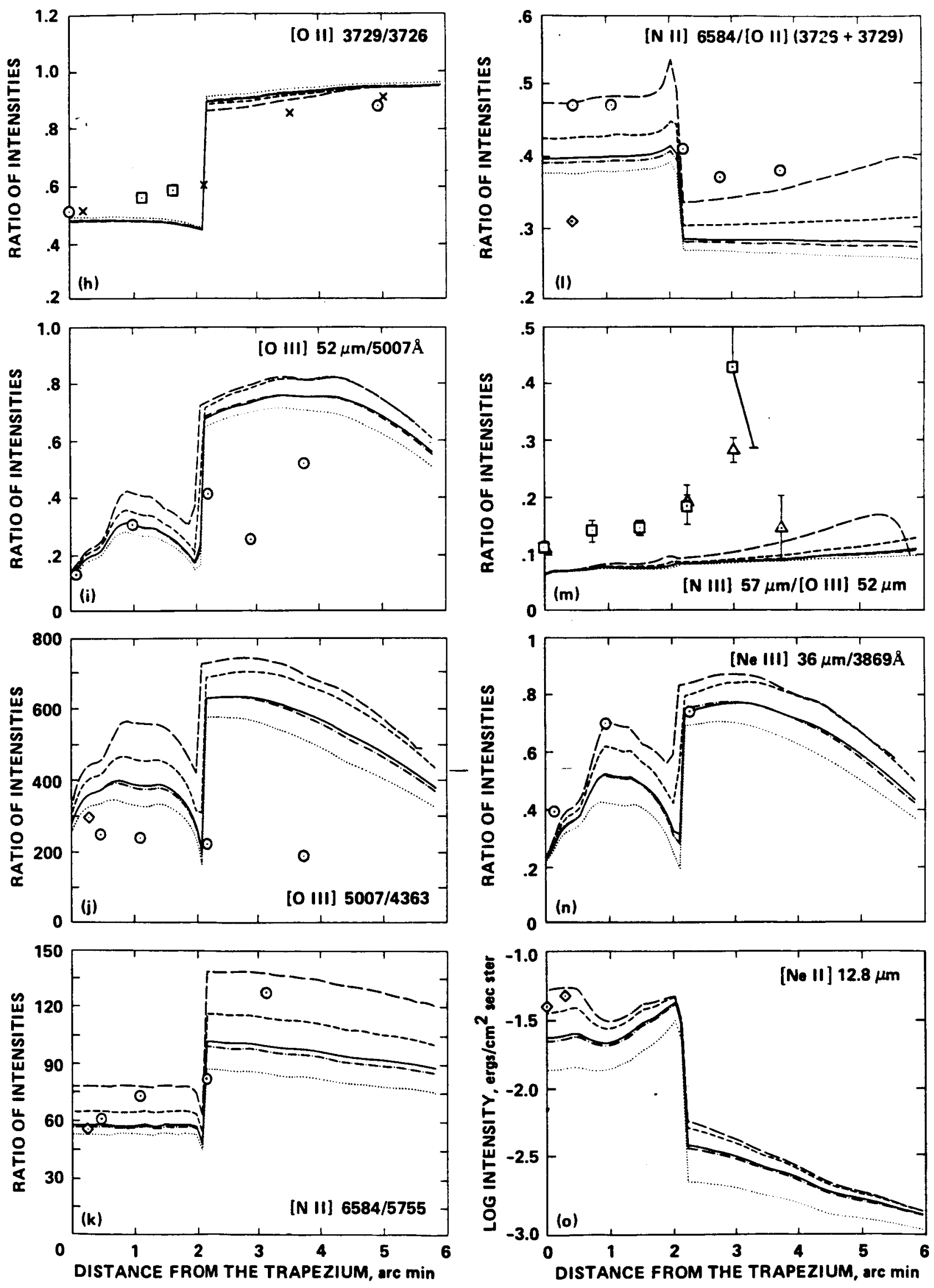


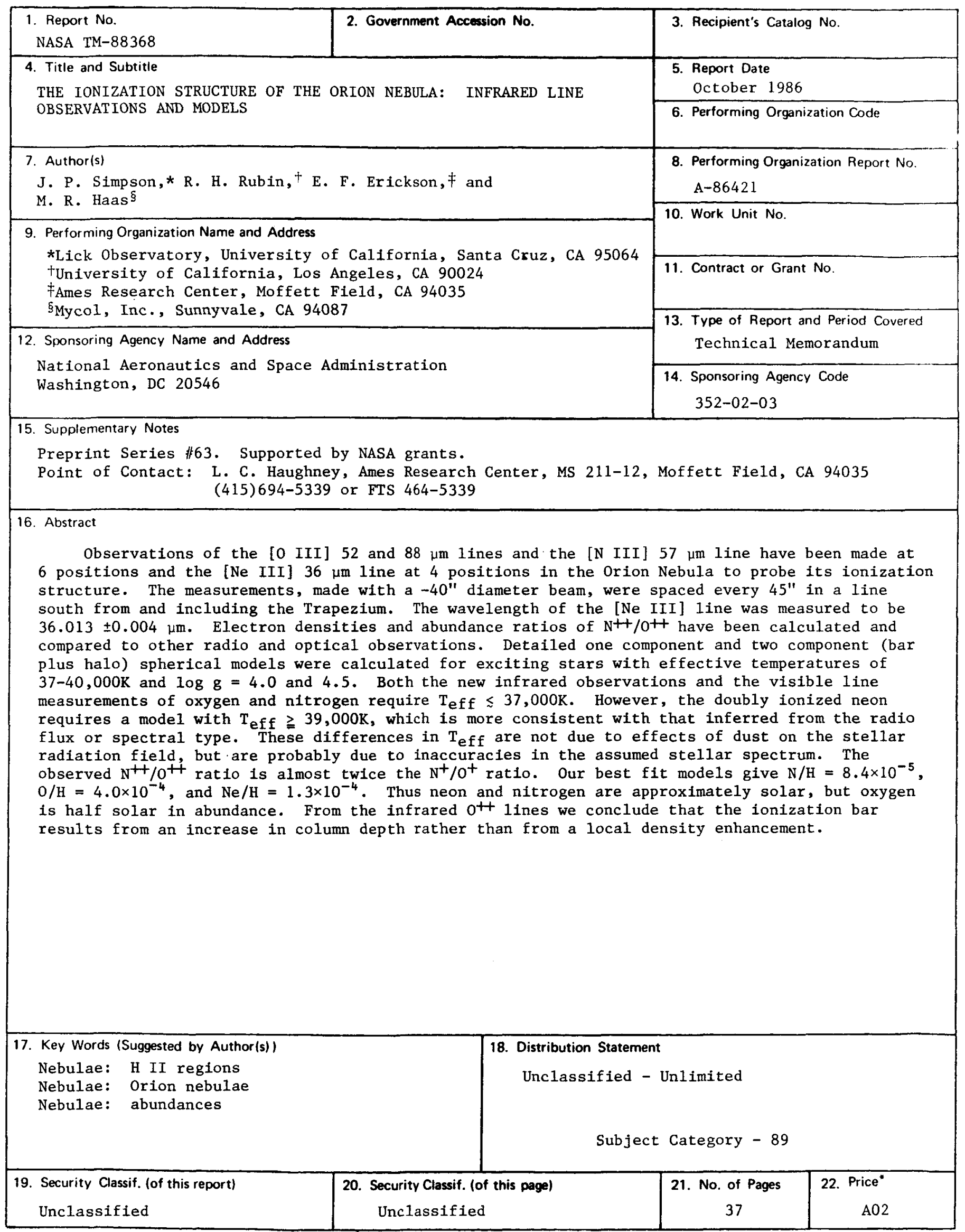

•For sale by the National Technical Information Service, Springfield, Virginia 22161 